\title{
Product Evaluation System Design of User Interaction Experience Based on the Concept of MDS
}

\author{
Xiaoyan Wang ${ }^{1, \text { a }}$, Aoge $\mathrm{Hu}^{1, \mathrm{~b}}$ and Yantao Zhong ${ }^{2, \mathrm{c}}$ \\ ${ }^{1}$ College of Mechanical and Electrical Engineering, Hohai University, Changzhou, China, 213022 \\ Academy of art Soochow University, Suzhou, China, 215123 \\ ${ }^{2}$ Department of art Changzhou University, Changzhou , China, 213164 \\ awxy826@126.com, b373785266@qq.com, czyt221@126.com
}

\begin{abstract}
Keywords: product design; interaction; evaluation system; product development
Abstract. Based on the concept of MDS, the interactive evaluation system is established to help the process of product development among products, dialogue between product and user, understanding, building a hierarchy, the level of cognition. Through the setting of cognitive standard, single dimension, basic dimension, information dimension, the snowflake design multi-dimensional integration, it is able to integrate the construction of interaction evaluation in the product development design. From the interactive practice development analysis of multidimensional evaluation models of different user observation models in 3 countries, it makes design develop in the direction of digital interaction, multimedia integrated use by the comprehensive, multi-angle interactive evalution methods..
\end{abstract}

\section{Introduction}

In the current competitive stage, products should pay more attention to the experience service through the interactive product evaluation system in the product design and development process. Product development and design process comes from concept analysis to model design to detailed design concept stage, and then enter the selection decision stage to a comprehensive assessment of the best program. The role of product evaluation is to select the continued or the terminated design, and the winner is able to go to the new product formulation stage, the evaluation system is an important process to determine the merits of the design but this phase is always ignored in the actual design development. Many outstanding, design-centered corporations have their own rating system or evaluation criteria to compare and evaluate the design development.

\section{MDS cognitive interaction concept}

\section{Origin of the MDS}

MD originates from the Multidimensional Scale, and it is also called Multidimensional Scaling. The current product evaluation systems have psychological design evaluation, aesthetic design evaluation, sensory evaluation, the evaluation of multidimensional scaling analysis can reflect the levels, reflecting that the level of interactive product experience cognitive assessment system can better improve the systematization of the product evaluation system without complicated formulas to demonstrate the evaluation in an operation, intuitive, fast, accurate, multi-faceted way. Generation and data acquisition and analysis in multidimensional purpose artificial construct, the specific interaction of cognitive completed by the organic combination of common carrier on all products, the formation of statistical analysis techniques with interactive ability to communicate in a particular context is multi-dimensional concept.

The establishment of the Multi-dimensional interaction of cognitive assessment system originates from 3 methods: One is the concept of product semantics which was formally put forward by the couple Krippendorff and Ho Lon Nol in 1983, and their main experiment is the theory about product forms and symbols indicate relations, Multidimensional scaling is a recognition method to compare 
product semantics, MDS will be converted into distance product semantics to identify more facets space. The second is a form location map technology co-founded by a professor at Carnegie Mellon University, School of Design and Engineering Professor Craig Vogel and Professor Jonathan Cogan from College of Engineering, The form location map shows the same field of different technologies and products in the form of an axis in the coordinate system of location; The third is the cost-benefit analysis funded by American scientist Benjamin Franklin, it will list the advantages and disadvantages of each program, and every advantage and disadvantage will be given 0 to $+10,00$ to -10 rating, and then add every pros and cons scores together, so you can come out of each program to determine which one is the best solution.

The concept of the product evaluation concept originated in concept of a consumer satisfaction by a consumer psychologist American. In contemporary style with statistical data in the form of a product evaluation score in the five-dimensional product design principles FACEBOOK, the principle is based on the user experience, five evaluation criteria for design evaluation score, then get valuable guidance.

The structure meaning of the MDS evaluation system

Span: Multi-level, multi-dimensional evaluation system provides more comprehensive, accurate and detailed data for reference for product itself, and provide multi-dimensional understanding of the product, multi-dimensional view of product design with a broader focus on the design dimensions, rational analysis and multidimensional analysis to better determine the direction of the product, the basic framework of creativity. Depth: It make goods and services more spiritual, so cold products have emotional functions to impress consumers in a distinguish direction, doing basic logical data, emotional communication between users and establish multi-dimensional product identification to create a common product and user experience emotional values.

Interactive multi-dimensional concept of cognition on the basis of statistics focus more multi-faceted, multi-angle construct, multidimensional look to evaluate the product, making it more dynamic and vitality. Multidimensional construct includes a combination of rational and emotional pluralistic structure of the visual language of form and emotion modeling capabilities beyond product data analysis capabilities, making product design development more feasible and practical significance, and thus doing a more comprehensive evaluation of product design and better to carry out product design development.

\section{The structure design of the cognitive interaction of MDS}

Interactive multi-dimensional structure of the design process of cognition can be summarized as "cognitive standard setting, single-dimensional architectural design, architecture design basis dimension, a dimension of design information architecture, multi-dimensional structure of a snowflake design", multi-dimensional structural design logic-oriented concepts from linear, pyramid-shaped, curved, grid layout logic, from global to local, simple to complex multi-dimensional thinking and cognitive structural product evaluation system, the following describes each process of design expression detailed separately.

Setting of the cognitive standard

Standards are designed to be fair to cognize design, cognitive standard positioning regardless of good or bad, regardless of right or wrong, its aim is to judge product design effectively. Whether designers, user groups, customer data, they are always having their own subjective consciousness and one-sided understanding of the establishment of standards, only exclude self-centered way of thinking cognitive get is real and feasible, and feedback to the designers, user groups, customer satisfaction has a fair measurement. The establishment of cognitive standards is the first step in the use of products and user experience cognitive interaction, and it is also the most crucial step. Awareness of the standard type setting is directly related to the establishment of the entire multi-dimensional evaluation system. 
Cognitive standard setting mainly refers to the following main types of cognitive: Emotion cognition is emotional awareness of product design mainly refers to the product caused by positive and negative emotions; Color cognition is easy to understand, that refers to the color of the match, the role of human emotions and psychological comfort level of the interface; function cognition mainly refers to the operation, comfort, controllability, coordination of the operation of products; Sense cognition mainly refers to the brightness of the product, gloss, texture, delicate sense, hard and soft surfaces, fine, cold flu of products; Material cognition mainly refers to product technology knowledge, identity, smoothness of the material, gloss, flexibility, cognitive contact, a single material and material combinations, such as the effectiveness of the distinction. For example, the standard set of cognitive interaction of FACEBOOK has five aspects: emotion, easy to use, form, the momentum, innovation, and the five indicators are divided into a total of twenty indicators of four aspects, each indicator by $0-5$ percentage points system scoring criteria to judge online user survey points and $50 \%$ in the form of a monthly review.

Structural design of a single dimension

Single-dimensional structure uses linear logic way, it is clear and targeted. Dimensional structure of one single dimension is mainly assembled by a dimension table, also known as zero-dimensional structures, in this structure, each dimension are based on points directly in the form of a rough product evaluation criteria evaluation, You can use the + and - or? To plus or minus, the best solution comes from the highest value of the product. The $\mathrm{ABC}$ three products in Figure 1 is evaluated by eight evaluation criteria as the basis for the single dimension of a rough assessment, those which meet the criteria are represented by + , those which break the criteria are represented by - and others are represents by filling? Finally, the highest score of 8 points is $C$ program, indicating that $C$ is the best option of three products. Single-dimensional structure is mainly focused on the current instinctive level which is the evaluation form of the appearance of product.

Structural design of the basis dimension - Get behavioral information

Structural design of the basis dimension uses pyramid logically, closely interrelated with each other. Dimensions are built by a two-dimensional design positioning based on statistical data base system, product positioning will analysis in detailed on the basis of cognitive criteria set. Underlying the product methodology in the basic dimension, it is to explore the interaction of various factors cognitive mutual restraint, influence, balanced relationship. Basic dimensions of structural design requires the use of information from the evaluation criteria to define the dimensions, each dimension of structure is to through the relationship between the trunk and branches directly linked to the fact table dimension table. FACEBOOK evaluation system in figure 2 is structured by the trunk to the branches, five major evaluation criteria is divided of four small items, such as major items under the precise positioning of emotion, affinity, warm nostalgia of the four small items, mainly from the behavior of the underlying dimension structure that level of product features, easy to understand, feel, usability evaluation of the details of the direction of behavior.

Structural design of the Information Dimensions_-Get information awareness

Information dimensions use a grid-type, logical curved way to achieve layout stability. Information dimension can be integrated two-dimensional or multi-dimensional database. Information dimension is based on property group product evaluation data source time, name, location, etc. list or view. Independent of the dimension of the cube can be used in the presence of either a two-dimensional data, it can also be used multiple times in cubes.

Information dimensions are divided into two aspects: 1) One is a two-dimensional point of view in the form of a cross-shaped area, each of the evaluation criteria is instructed of 5 points, when a closed space area is larger, the higher the score, as shown in Apple's iPod nano and iPod photo, they are achieved by the establishment cross-type coordinate dimension, the appearance, ease of use, price and function are evaluated by 1-5 point, there is a big difference between the two products in terms of price and features two dimensions of evaluation, the more expensive iPod photo has less function. At last, iPod nano wins a litter with a larger area to locate edge; 2) The second is to deduct data interpretation according to line and grid, the structure of product awareness is quantified by the peak 
line or grid arrangement embodied in the form of guidance, the curve of the upper and lower peak is no better or worse, it is based on product perception measurement in the large cognitive data to qualitative indicators. By fluctuations in the peak value, the cognitive perception of each product is deducted by design. Information dimensional structure is evaluated mainly from the level of consciousness: product of business, innovation and experience.

Multidimensional Structural design—_-Get Subliminal information

Multi-dimensional structure design uses spider web in a logic way which has rich layers and careful thinking. Multi-dimensional structure of the information need many charts to define, and finally merged to form integrated dimension to show the overall evaluation, the general evaluation criteria is used as branch of structure to show all products of factors such as data processing for the crowd, cognitive scope, culture background, gender differences to summarize the acceptance of product information as well as people's way of thinking and habits.

Spatial dimension of the visual language of form can construct out of the product form, emotional, professional and other full-frame multiple attributes, reflecting cognitive scores of user groups more directly. Spatial dimensions from macro to micro, from micro to analysis quantitatively, using spiderweb-like multi-faceted type of cognitive structure as a major integrated visual manifestation. In the cognitive process, we should use the concept of big data to establish numerical structure diagram instead of precise data to integrate the integration of perceptual awareness of each product. In the multidimensional structure design of FACEBOOK in figure 4, "easy to use" gets the highest score and "beauty in shape" followed, "innovation" gets the lowest score and "emotion" followed by, we can see that "copy" gets the lowest scores of four small items of innovative observation score. In the user's subconscious, we need to focus on discrimination of the product with other similar products in order to make product have a vigorous vitality, and FACEBOOK's praise button design is a good example.

\section{Conclusions}

MDS multidimensional interaction of cognitive evaluation system achieved a comprehensive evaluation of product design from the instinctive level, behavioral level to the level of reflection to understand users' unfilled needs and unmet needs. It is able to do the analysis of product development cognitive interaction from product concept essences and scientific concept. It is not in pursuit of innovation and change for the guidelines, and to enhance the stability analysis as a benchmark for change product interaction, so that visual information dissemination product modeling transition occurs in content and form, and then develops into a multi-level, comprehensive modeling language. Emotional, conceptual design ideas are survive and add with digital cognitively interaction to achieve a leap from static product modeling to interactive forms to make the expression of product interactive cognition more plumply and smoothly.

\section{References}

[1] Li Zhang, Wei Li, Creative graphics and creative thinking, Changsha: Hunan arts publishing house, 2003. (In Chinese)

[2] Dong, Y., Lee, K. P. (2008). A cross-cultural comparative study of users' perceptions of a webpage: With a focus on the cognitive styles of Chinese, Koreans and Americans. International Journal of Design, 2(2), 19-30.

[3] Chen, S. J, \& Ford, N. (1998). Modeling user navigation behaviors in a hypermedia-based learning system: An individual differences approach. International Journal of Knowledge Organization, 25(3), 67-78. 